\title{
BMJ Open Trends of prescribing adherence of antiplatelet agents in Hong Kong patients with acute coronary syndrome: a 10-year retrospective observational cohort study
}

Amy SM Lam (D) , ${ }^{1}$ Bryan PY Yan, ${ }^{1}$ Vivian WY Lee (D) ${ }^{2}$

To cite: Lam ASM, Yan BPY, Lee VWY. Trends of prescribing adherence of antiplatelet agents in Hong Kong patients with acute coronary syndrome: a 10year retrospective observational cohort study. BMJ Open 2020;10:e042229. doi:10.1136/ bmjopen-2020-042229

- Prepublication history and additional material for this paper is available online. To view these files, please visit the journal online (http://dx.doi.org/10. 1136/bmjopen-2020-042229).

Received 29 June 2020 Revised 16 November 2020 Accepted 23 November 2020

Check for updates

(c) Author(s) (or their employer(s)) 2020. Re-use permitted under CC BY-NC. No commercial re-use. See rights and permissions. Published by BMJ.

${ }^{1}$ Department of Medicine and Therapeutics, Faculty of Medicine, The Chinese University of Hong Kong, Hong Kong, Hong Kong

${ }^{2}$ Centre for Learning

Enhancement And Research, The Chinese University of Hong Kong, Hong Kong, Hong Kong

Correspondence to

Dr Vivian WY Lee;

vivianlee@cuhk.edu.hk

\section{ABSTRACT}

Objectives The objective of this study is to examine the temporal trend of antiplatelet prescribing pattern during index hospitalisation discharge in Hong Kong (HK) acute coronary syndrome (ACS) population.

Design The study is a retrospective observational cohort study.

Setting The study retrieved data from electronic health record from Hospital Authority (HA), HK.

Participants The study included patients aged 18 years old or above, who were admitted to seven institutions under HA with diagnosis of ACS during 2008-2017.

Primary and secondary outcome measures The primary outcome was the frequency of antiplatelet therapy prescription at the point of index hospitalisation discharge each year during 2008-2017. Association between demographics, baseline comorbidities, procedures and antiplatelet prescription were examined as secondary outcome using multivariate logistic regression model, with commonly used antiplatelet groups selected for comparison.

Results Among the included 14716 patients, 5888 (40.0\%) discharged with aspirin alone, 6888 (46.8\%) discharged with dual antiplatelet therapy (DAPT) with clopidogrel, and 973 (6.6\%) discharged with DAPT with prasugrel/ticagrelor. Prescribing rate of aspirin alone decreased substantially from $56.8 \%$ in 2008 to $27.5 \%$ in 2017. Utilisation of DAPT with clopidogrel increased from $33.7 \%$ in 2008 to $52.7 \%$ in 2017 . Use of DAPT with prasugrel/ticagrelor increased from $0.3 \%$ in 2010 to $15.3 \%$ in 2017. Compared with those prescribed with DAPT with clopidogrel, male patients (adjusted OR (aOR) $1.34,95 \% \mathrm{Cl} 1.09$ to 1.65 ), patients with non-ST-elevation myocardial infarction (aOR 2.50, 1.98 to 3.16) or STelevation myocardial infarction (aOR 3.26, 2.59 to 4.09), use of glycoprotein Ilb/llla (aOR 3.03, 2.48 to 3.68) or undergoing percutaneous coronary intervention (aOR 3.85 , 3.24 to 4.58 ) or coronary artery bypass graft (aOR 6.52 , 4.63 to 9.18 ) during index hospitalisation, concurrent use of histamine-2 receptor antagonists (aOR 1.35, 1.10 to 1.65) or proton pump inhibitors (aOR $3.57,2.93$ to 4.36 ) during index hospitalisation discharge were more likely to be prescribed with DAPT with prasugrel/ticagrelor. Patients with older age (aOR 0.97, 0.96 to 0.97), diabetes (aOR $0.68,0.52$ to 0.88 ), chronic kidney disease (aOR 0.43 ,
Strengths and limitations of this study

- This is the first study to investigate 10 -year antiplatelet prescription pattern in patients with acute coronary syndrome in Hong Kong.

- The retrospective real-world observational study collected clinical data through territory-wide electronic health record system.

- The study showed slow adaption on use of newer P2Y12 inhibitor in Hong Kong, and association between conservative treatment strategy and identified risk factors for bleeding.

- The study did not account for availability of medication in individual institution and patients' preference.

0.22 to 0.85 ) or concurrent use of oral anticoagulant (aOR $0.16,0.07$ to 0.42 ) were more likely to received DAPT with clopidogrel.

Conclusions Use of DAPT with prasugrel/ticagrelor was suboptimal yet improving during 2008-2017 in HK patients with ACS. Considering DAPT, predictors for clopidogrel prescription, compared with prasugrel/ ticagrelor, were consistent with identified risk factors of bleeding.

\section{INTRODUCTION}

Use of dual antiplatelet therapy (DAPT) in patients with acute coronary syndrome (ACS) has been introduced since 2002. Clinical benefit on major adverse cardiovascular (CV) event reduction of DAPT has been shown in numerous studies. ${ }^{1-4}$ Recommendations from clinical practice guidelines advocates use of DAPT over aspirin alone. ${ }^{56}$ Prasugrel and ticagrelor have been introduced to the market for 10 years. Additional clinical benefits shown in landmark trials supported the guidelines' update on choice of P2Y12 receptor antagonists accordingly. The Trial to Assess Improvement in Therapeutic Outcomes by Optimizing Platelet Inhibition 
With Prasugrel-Thrombolysis in Myocardial Infarction 38 (TRITON-TIMI 38) demonstrated that prasugrel reduced risk of $\mathrm{CV}$ death, non-fatal myocardial infarction (MI) or non-fatal stroke in patients with ACS with percutaneous coronary intervention (PCI), compared with clopidogrel. ${ }^{7}$ Clinical benefit of ticagrelor over clopidogrel in patients with ACS was established in the PLATO trial, which showed risk reduction in death from vascular causes, MI or stroke. ${ }^{8}$ Clinical guideline recommended use of ticagrelor over clopidogrel in patient with ACS received medical care without revascularisation, while prasugrel or ticagrelor was preferred over clopidogrel in post-PCI patients with ACS. ${ }^{9}$

Suboptimal DAPT adherence in ACS population was observed in published literature from other countries. Prevalence of DAPT use in patients with ACS varied from $67 \%$ to $87 \% .^{10-12}$ Use of DAPT was reported even lower in patients with ACS without receiving revascularisation, ranging from $44 \%$ to $81.8 \% .{ }^{101113}$ Use of prasugrel or ticagrelor in patients with ACS ranged from $8 \%$ to $49.8 \% .{ }^{12} 14$ Yet comprehensive description on current antiplatelet prescribing strategy in Hong Kong patients with ACS is lacking. With the findings on antiplatelet prescription pattern, it is hoped to review the guideline recommendation adaptation by local healthcare practitioners and to provide evidence for local healthcare policy-makers for future prescription policy planning and implementation.

The primary objective of this study was to describe the frequency of antiplatelet therapy prescription at the point of index hospitalisation discharge each year from 2008 to 2017 using electronic health records. Association between demographics, baseline comorbidities, procedures and antiplatelet prescription are examined as secondary objectives, with commonly used antiplatelet groups selected for comparison.

\section{METHODOLOGY}

\section{Study design and study population}

This real-world retrospective territory-wide observational cohort study included patients, aged 18 years old or above, admitted to seven institutions under New Territories East cluster (NTEC), Hospital Authority (HA), Hong Kong, of which serves the districts of 1.31 million population in total, with diagnosis of ACS from 1 January 2008 to 31 December 2017. ${ }^{15}$ The seven institutions included three acute hospital (Prince of Wales Hospital, Alice Ho Miu Ling Nethersole Hospital, North District Hospital), three extended-care hospitals (Shatin Hospital, Tai Po Hospital, Cheshire Home Sha Tin) and a hospice (Bradbury Hospice). Being the largest healthcare providers in Hong Kong, HA managed $85.9 \%$ of all hospital beds in Hong Kong as of 2017 (excluding nursing homes and correctional institution). ${ }^{16}$ In particular, $96 \%$ of discharges and deaths related to acute MI were from hospitals under HA. ${ }^{17}$ Demographic, clinical and procedural data and drug dispensing history were retrieved through HA clinical data analysis and report system (CDARS). Quality of data from CDARS was assessed in previous study, showing CDARS being a valid tool for research purpose with high positive predictive value in identifying diagnosis $(96.8 \%) .{ }^{18}$ Admission diagnosis, baseline demographics and comorbidities, and procedures were identified based on International Classification of Disease, 9th Revision Clinical Modification (ICD-9-CM) codes. List of ICD-9-CM codes used was attached in supplementary document (table s1). Information on demographics and comorbidities included sex, age on admission, hypertension, diabetes, heart failure, chronic kidney disease, liver disease, arrhythmia, dyslipidaemia, history of stroke (ischaemic or haemorrhagic), ischaemic heart disease, history of ACS episode, anaemia or history of bleeding event. Information on procedures included PCI with bare metal stent, PCI with drug-eluting stent and coronary artery bypass graft $(\mathrm{CABG})$. Concurrent prescription of oral anticoagulant (OAC), including warfarin, dabigatran, rivaroxaban, apixaban and edoxaban, gastric protectants including histamine-2 receptor antagonists (H2RA) and proton pump inhibitors (PPI), use of fibrinolytics or glycoprotein IIb/IIIa (GP IIb/IIIa) receptor inhibitors during index hospitalisation were also captured.

Focus of current study is on maintenance phase of antiplatelet therapy, instead of acute antiplatelet treatment. Patients who died at index hospitalisation or missing drug dispensing record were excluded. Antiplatelet prescription was defined as antiplatelet agent prescribed during index hospitalisation discharge. Antiplatelet use only during index hospitalisation yet not continued on discharge was not described in this study. Subjects were categorised into groups of 'Aspirin only', 'Clopidogrel only', 'Prasugrel or Ticagrelor only', 'DAPT with clopidogrel', 'DAPT with Prasugrel', 'DAPT with Ticagrelor', 'Other' and 'No antiplatelet'. 'Other' group was defined as concurrent prescription of prasugrel and ticagrelor.

\section{Subgroup analysis}

Choice of antiplatelet depends on ACS type and status of revascularisation. Concurrent use of antiplatelet and OAC is indicated in atrial fibrillation patients with ACS undergoing PCI. ${ }^{19}$ This strategy increases risk of bleeding, which could affect choice of antiplatelet agent. ${ }^{20}$ Subgroup analyses of description on antiplatelet prescription and association between demographics, baseline comorbidities, procedures and antiplatelet prescription were done in (1) patients undergoing PCI during index hospitalisation; (2) patients receiving medical therapy only during index hospitalisation admission; and (3) patients with concurrent prescription of antiplatelet and OAC at the point of index hospitalisation discharge.

As clinical practice guidelines updated along the time, change of real-world practice was expected accordingly. Ticagrelor was first introduced in 2012 American College of Cardiology Foundation/American Heart Association (ACCF/AHA) clinical practice guideline in non-ST-segment elevation (NSTE)-ACS management, while preferred use of newer P2Y12 receptor 
antagonists was stated in clinical practice guideline update in $2014 .^{521}$ Therefore, further subgroup analysis on association between demographics, baseline comorbidities, procedures and antiplatelet prescription was done in patients admitted after 2014 to test robustness of previous results.

\section{Statistical analysis}

Descriptive statistics were presented as counts (percentage) for qualitative variable and mean (SD) for quantitative variable. Comparison on qualitative variables was made between groups with Pearson's $\chi^{2}$ test or Fisher's exact test if appropriate. ${ }^{22}$ Difference on quantitative variables between groups was compared with Student's t-test. As multiple testing was adopted with the existence of more than two groups, $p$ value was adjusted using the Benjamini and Hochberg method. Association of antiplatelet prescription preference and variables was examined using both univariate and multivariate logistic regression and presented with OR, adjusted OR (aOR) and $95 \%$ CI. Demographics, baseline comorbidities, admission diagnosis, procedures during index hospitalisation and concurrent anticoagulant use were included in variable selection process. Stepwise selection was used in logistic regression model building. Statistical signifanalyses were performed using R (V.3.5.3).

\section{Patient and public involvement}

This research was done without patient involvement. Patients were not invited to comment on the study design and were not consulted to develop patient relevant outcomes or interpret the results. Patients were not invited to contribute to the writing or editing of this document for readability or accuracy. icance was defined as $\mathrm{p}<0.05$ (two sided). All statistical

\section{RESULTS}

A total number of 17893 patients aged 18 years old or above was admitted to HA NTEC hospital for ACS during 2008-2017. Of these 17893 patients, 3127 subjects were excluded due to death during index hospitalisation, and 50 were excluded due to missing drug dispensing record. Among the included cohort of 14716 subjects, 5888 $(40.0 \%)$ of subjects were prescribed with aspirin alone, $173(1.2 \%)$ with clopidogrel alone, $7(<0.05 \%)$ with prasugrel or ticagrelor alone, $6888(46.8 \%)$ with DAPT with clopidogrel, $123(0.8 \%)$ with DAPT with prasugrel, 850 $(5.8 \%)$ with DAPT with ticagrelor, 783 (5.3\%) without any antiplatelet agents and $4(<0.05 \%)$ with concurrent dispensing record of prasugrel and ticagrelor when discharged. There were $7861(53.4 \%)$ prescribed with DAPT at the index hospitalisation discharge over the 10-year cohort. Prescription of prasugrel in patients with ACS was first detected in 2010, while that of ticagrelor was in 2012. The study cohort diagram was shown in figure 1 . Demographics and baseline comorbidities of overall cohort, groups of aspirin only, DAPT with clopidogrel, DAPT with prasugrel or ticagrelor and no antiplatelet, were described in table 1.

Trend of antiplatelet prescription for each year during 2008-2017 was shown in table 2 and illustrated in figure 2. Common antiplatelet treatment in patients with post-ACS included aspirin only and DAPT with clopidogrel from 2008 to 2017. Use of aspirin only as antiplatelet treatment in patients with post-ACS was substantially decreased from $56.8 \%$ in 2008 to $27.5 \%$ in 2017 . On the contrary, use of DAPT with clopidogrel was increased from $33.7 \%$ in 2008, peaked at $55.7 \%$ in 2012, and remained stable at approximately $50 \%$ in subsequent years. Focusing on newer P2Y12 receptor antagonists, use of prasugrel as DAPT in Hong Kong remained unpopular in Hong Kong throughout. Since 2012, use of ticagrelor has been increased from $2.7 \%$ to $16.8 \%$ in 2016 and was maintained at $15.3 \%$ in 2017 . When we considered the overall use of

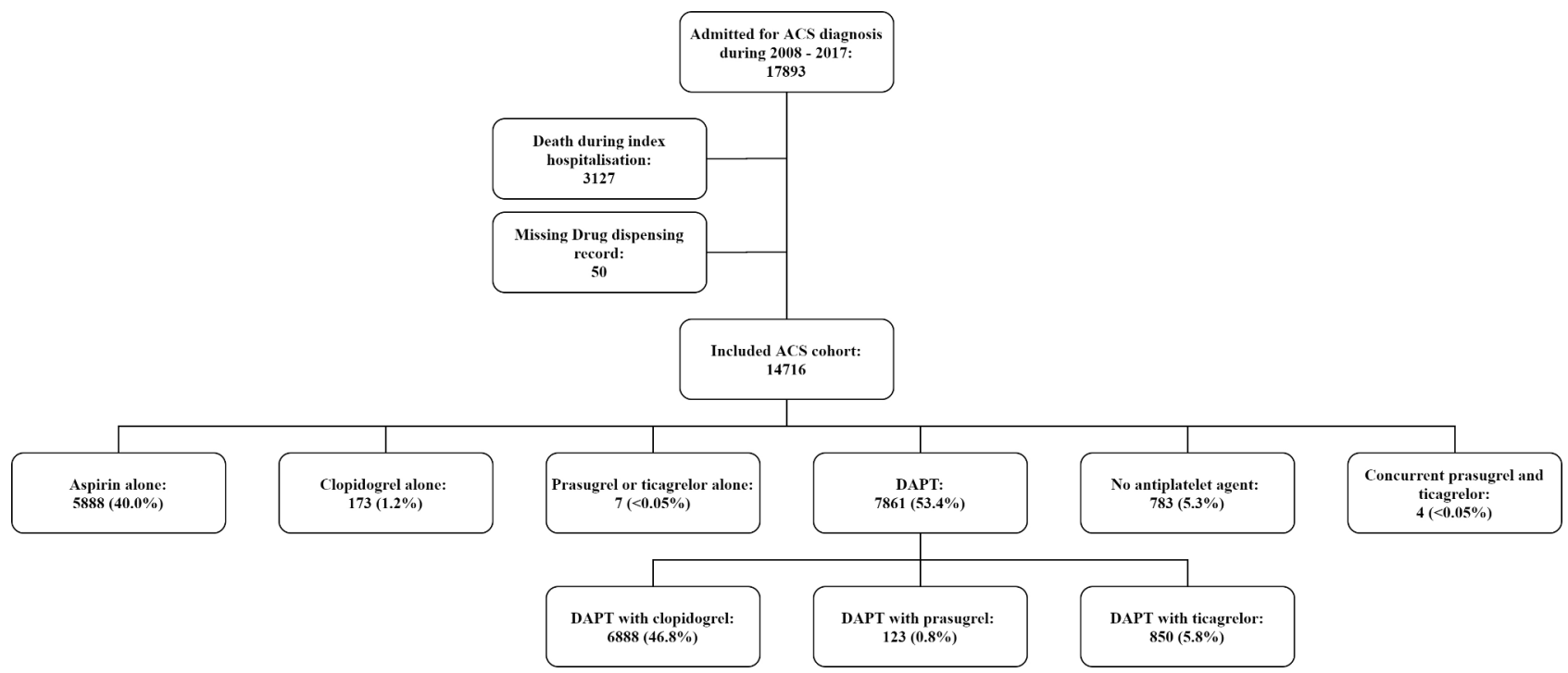

Figure 1 Study cohort diagram for the analysis. ACS, acute coronary syndrome. DAPT, dual antiplatelet therapy. 
Table 1 Demographics and baseline comorbidities of overall cohort, treatment groups of aspirin only, dual antiplatelet therapy (DAPT) with clopidogrel, DAPT with prasugrel or ticagrelor, and no antiplatelet

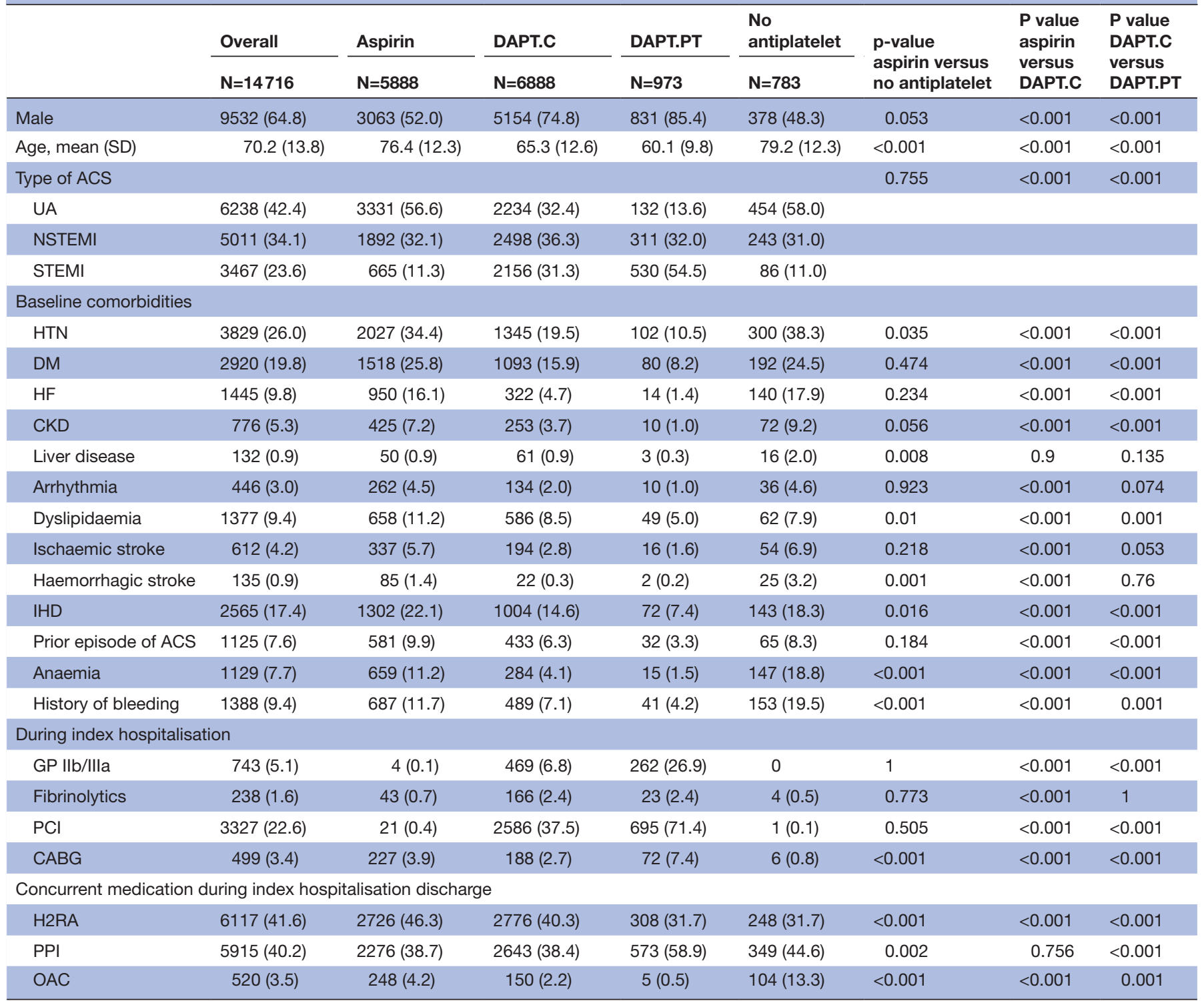

Data are shown as frequency (percentage) unless specified. Multiple comparison was done among the presented groups. Only adjusted p value for comparison between (1) aspirin and no antiplatelet group; (2) aspirin and DAPT with clopidogrel group; and (3) DAPT with clopidogrel and DAPT with prasugrel or ticagrelor, were shown.

ACS, acute coronary syndrome; CABG, coronary artery bypass grafting; CKD, chronic kidney disease; DAPT.C, dual antiplatelet therapy with clopidogrel; DAPT. $\mathrm{PT}$, dual antiplatelet therapy with prasugrel or ticagrelor; DM, diabetes; HF, heart failure; H2RA, histamine-2 receptor antagonist; HTN, hypertension; IHD, ischaemic heart disease; GP Ilb/Illa, glycoprotein Ilb/IIla receptor inhibitor; NSTEMI, non-ST-elevation myocardial infarction; OAC, oral anticoagulant; PCI, percutaneous coronary intervention; PPI, proton pump inhibitor; STEMI, ST-elevation myocardial infarction; UA, unstable angina.;

DAPT regardless of choice of P2Y12 receptor antagonist, the proportion of patients receiving DAPT was increased significantly from $33.7 \%$ in 2008 to $68.0 \%$ in 2017 .

Subgroup analysis considering PCI status and status of concurrent OAC use showed difference antiplatelet prescription pattern. Detailed results were shown in supplementary tables, respectively, on (1) patients with PCI done during index hospitalisation (online supplemental table s2 and figure s1); (2) patients received only medical care without revascularisation during index hospitalisation (online supplemental table s3 and figure s2); and (3) patients with concurrent OAC (online supplemental table s4 and figure s3).

Utilisation of DAPT was consistently above $95 \%$ in post-PCI patients with ACS ( $n=3327)$. Since the introduction of prasugrel and ticagrelor, proportional of patients receiving clopidogrel dropped to $59.7 \%$ of all DAPT users, while that of ticagrelor increased to $40.3 \%$ in 2017 . In patients received only medical care without revascularisation during index hospitalisation $(\mathrm{n}=11368)$, a large proportion of subjects received aspirin only as discharge antiplatelet treatment. The prescription rate of aspirin 


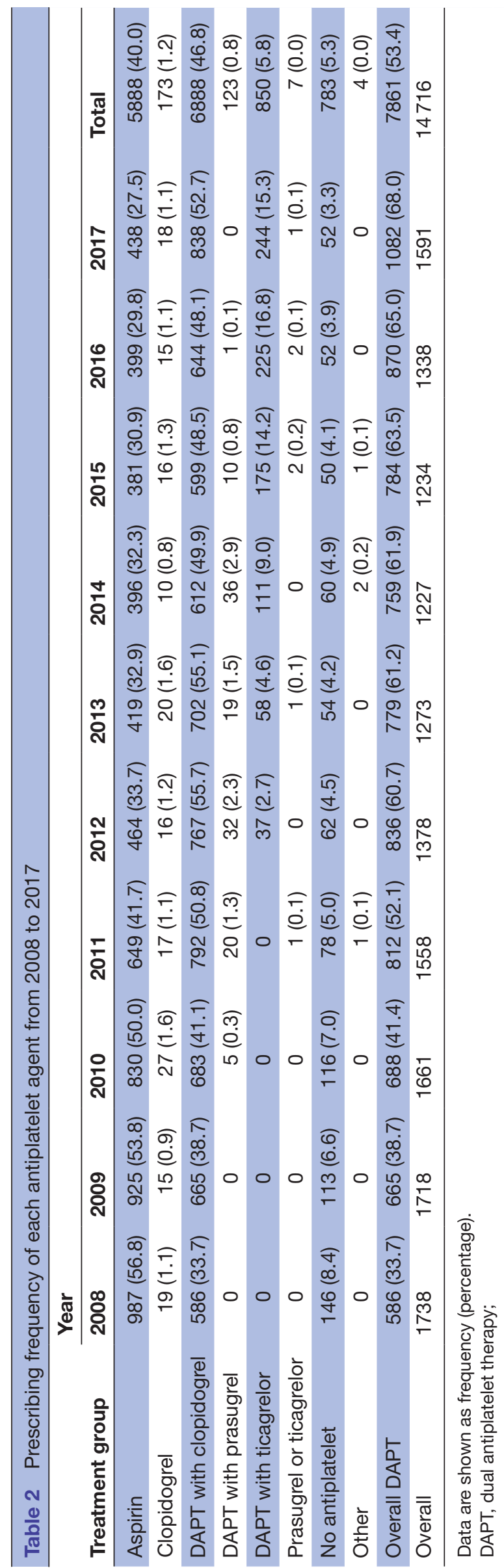

only decreased from $62.4 \%$ in 2008 to $44.5 \%$ in 2012 and remained stable with $39.3 \%$ in 2017. Strategy on use of aspirin switched to use of DAPT with clopidogrel, as we observed prescription rate of DAPT with clopidogrel increased from $27.4 \%$ in 2008 to $46.4 \%$ in 2012 and maintained at $49.8 \%$ in 2017 . Only a small proportion of subjects received DAPT with ticagrelor. A significant proportion of subjects was not receiving any antiplatelet agents at index hospitalisation discharge. The rate of 'no antiplatelet' was at $9.2 \%$ in 2008 , and slowly dropped to $4.7 \%$ in 2017.

A subgroup description on antiplatelet prescription pattern was done in patients prescribed with OAC during index hospitalisation discharge. A total number of 520 subjects (3.5\% of overall included cohort) were included in this subgroup analysis. Majority of the group was prescribed with aspirin only $(47.7 \%)$, followed by DAPT with clopidogrel $(28.8 \%)$ and no antiplatelet prescribed $(20.0 \%)$. Use of DAPT with clopidogrel with concurrent OAC was increased from $18.8 \%$ in 2008 to $36.8 \%$ in 2017 , while 'no antiplatelet' dropped from $26.6 \%$ in 2008 to $13.2 \%$ in 2017. To highlight, the rate of 'no antiplatelet' group was the highest in the subgroup of patients with concurrent OAC use, compared with other subgroups.

When comparing DAPT with clopidogrel and aspirin alone groups, patients with older age (aOR 0.94, 95\% CI 0.94 to 0.95 ), diabetes (aOR $0.78,95 \%$ CI 0.70 to 0.88 ), heart failure (aOR $0.60,95 \%$ CI 0.51 to 0.71 ), chronic kidney disease (aOR $0.74,95 \%$ CI 0.60 to 0.90 ), history of haemorrhagic stroke (aOR $0.36,95 \%$ CI 0.20 to 0.63 ), anaemia (aOR $0.65,95 \% \mathrm{CI} 0.54$ to 0.78 ), $\mathrm{CABG}$ (aOR $0.68,95 \%$ CI 0.54 to 0.86 ) during index hospitalisation, concurrent H2RA (aOR $0.88,95 \%$ CI 0.79 to 0.99 ) or OAC (aOR $0.40,95 \%$ CI 0.30 to 0.52 ) during index hospitalisation discharge, were more likely to be prescribed with aspirin alone (table 3). Male patients (aOR 1.33, 95\% CI 1.20 to 1.46 ), patients admitted for non-ST-elevation myocardial infarction (NSTEMI) (aOR 2.58, 95\% CI 2.33 to 2.86) or ST-elevation myocardial infarction (STEMI) (aOR 3.00, 95\% CI 2.64 to 3.40), baseline comorbidities of ischaemic heart disease (aOR 1.14, 95\% CI 1.01 to 1.29), use of GP IIb/IIIa (aOR 16.64, 95\% CI 5.91 to 46.81) or undergoing PCI (aOR 112.90, 95\% CI 73.05 to 174.51) during index hospitalisation, or concurrent use of PPI (aOR 1.32, 95\% CI 1.17 to 1.48 ) during index hospitalisation discharge, were more likely to be prescribed with DAPT with clopidogrel. When comparing DAPT with clopidogrel and DAPT with prasugrel or ticagrelor, patients with older age (aOR $0.97,95 \%$ CI 0.96 to 0.97 ), diabetes (aOR $0.68,95 \%$ CI 0.52 to 0.88 ), chronic kidney disease (aOR $0.43,95 \%$ CI 0.22 to 0.85 ) or concurrent use of OAC (aOR $0.16,95 \%$ CI 0.07 to 0.42 ) were more likely to received DAPT with clopidogrel (table 4, figure 3). Male patients (aOR 1.34, 95\% CI 1.09 to 1.65), patients with NSTEMI (aOR 2.50, 95\% CI 1.98 to 3.16) or STEMI (aOR 3.26, 95\% CI 2.59 to 4.09), use of GP IIb/IIIa (aOR $3.03,95 \%$ CI 2.48 to 3.68 ) or undergoing PCI (aOR 3.85, 
Antiplatelet Prescription Pattern from 2008 to 2017 in ACS patients

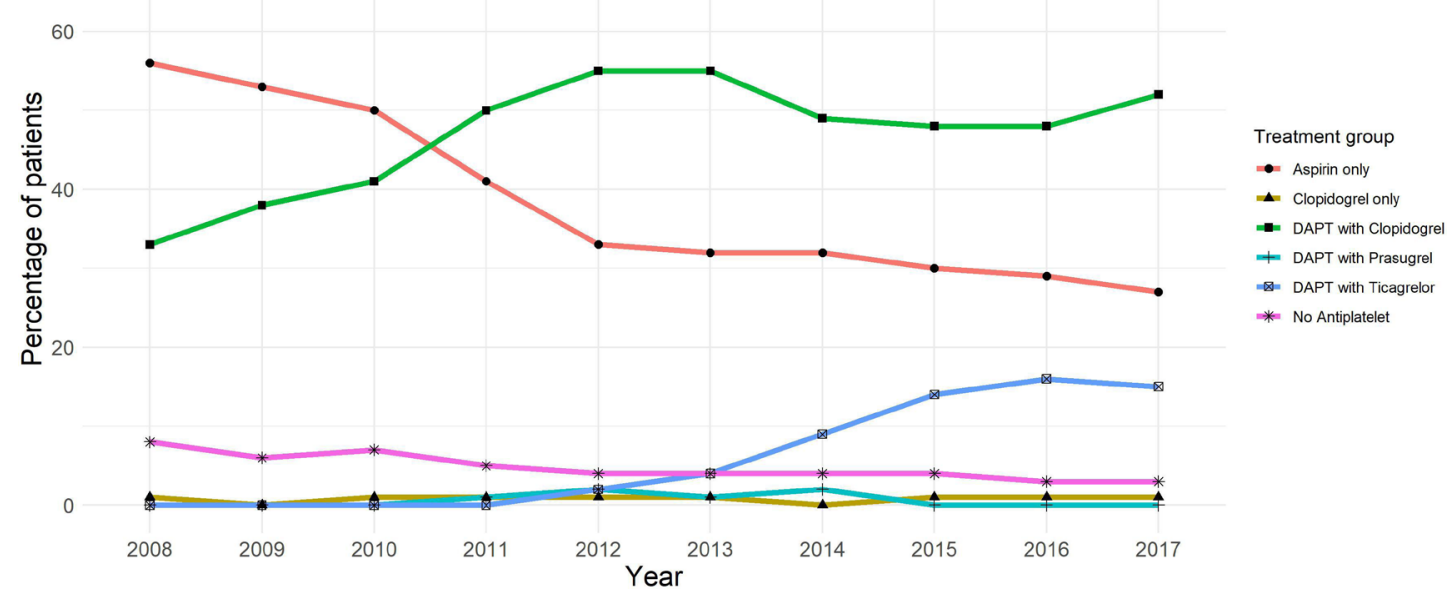

Figure 2 Prescription pattern of antiplatelet agents from 2008 to 2017. ACS, acute coronary syndrome. DAPT, dual antiplatelet therapy.

95\% CI 3.24 to 4.58 ) or CABG (aOR 6.52, 95\% CI 4.63 to 9.18) during index hospitalisation, concurrent use of H2RA (aOR $1.35,95 \%$ CI 1.10 to 1.65 ) or PPI (aOR $3.57,95 \%$ CI 2.93 to 4.36 ), during index hospitalisation discharge were more likely to be prescribed with DAPT with prasugrel or ticagrelor.

Subgroup analysis on association between demographics, baseline comorbidities, procedures and antiplatelet prescription was done in post-PCI patients (online supplemental table s5) and medically treated patients with ACS (online supplemental table s6), with details shown in online supplemental tables.

In post-PCI patients, DAPT with clopidogrel and DAPT with prasugrel and ticagrelor were selected for investigation. Patients with older age (aOR 0.97, 95\% CI 0.96 to 0.98 ), diabetes (aOR $0.56,95 \% \mathrm{CI} 0.39$ to 0.82 ), or concurrent use of OAC (aOR $0.08,95 \%$ CI 0.02 to 0.33 ), were more likely to be prescribed with DAPT with clopidogrel. Male patients (aOR 1.32, 95\% CI 1.02 to 1.71), patients admitted for NSTEMI (aOR 2.30, 95\% CI 1.71 to 3.09 ) or STEMI (aOR 2.95, 95\% CI 2.23 to 3.90), GP $\mathrm{IIb} / \mathrm{III}$ use (aOR 2.59, 95\% CI 2.08 to 3.22) during index hospitalisation, concurrent use of H2RA (aOR 1.32, $95 \%$ CI 1.03 to 1.70 ) or PPI (aOR $4.57,95 \%$ CI 3.57 to 5.85) during index hospitalisation discharge, were more likely to be prescribed with DAPT with prasugrel or ticagrelor. In medically treated patients with ACS, majority of patients were prescribed with DAPT with clopidogrel or aspirin alone. Patients with older age (aOR 0.94, $95 \%$ CI 0.94 to 0.95 ), diabetes (aOR $0.79,95 \%$ CI 0.70 to 0.89 ), heart failure (aOR $0.59,95 \%$ CI 0.50 to 0.70 ), chronic kidney disease (aOR $0.73,95 \%$ CI 0.59 to 0.89 ), history of ischaemic (aOR $0.80,95 \%$ CI 0.64 to 0.99 ) or haemorrhagic stroke (aOR $0.38,95 \% \mathrm{CI} 0.22$ to 0.68 ), anaemia (aOR 0.64, 95\% CI 0.53 to 0.77 ), undergoing CABG during index hospitalisation (aOR 0.69, 95\% CI 0.55 to 0.87 ), concurrent use of H2RA (aOR $0.88,95 \%$ CI
0.79 to 0.99 )_or OAC (aOR $0.39,95 \%$ CI 0.30 to 0.52 ) during index hospitalisation discharge, were more likely to be prescribed with aspirin alone. On the other hand, male patients (aOR 1.33, 95\% CI 1.21 to 1.47 ), patients admitted for NSTEMI (aOR 2.60, 95\% CI 2.34 to 2.89) or STEMI (aOR 3.05, 95\% CI 2.68 to 3.46), GP IIb/IIIa during index hospitalisation (aOR 21.77, 95\% CI 6.66 to 71.13), or concurrent use of PPI during index hospitalisation discharge (aOR 1.32, 95\% CI 1.17 to 1.49 ), were more likely to be prescribed with DAPT with clopidogrel.

An additional set of analyses on predictors for antiplatelet prescription in patients admitted during 2014 to 2017 was done with the following comparison groups: (1) overall cohort, DAPT with clopidogrel versus aspirin only; (2) overall cohort, DAPT with clopidogrel versus DAPT with prasugrel or ticagrelor; (3) post-PCI cohort, DAPT with clopidogrel versus DAPT with prasugrel or ticagrelor; (4) medically treated ACS cohort, DAPT with clopidogrel versus aspirin only.

In overall cohort comparing DAPT with clopidogrel and aspirin alone, most of the results on predictors remained robust. In the subgroup of 2014-2017 cohort, comorbidities of chronic kidney disease, ischaemic heart disease, use of GP IIb/IIIa, and concurrent use of PPI were no longer predictors for the choice of antiplatelet medication. On the contrary, ischaemic stroke (aOR $0.69,95 \%$ CI 0.49 to 0.98 ), prior episode of ACS (aOR 2.20, 95\% CI 1.53 to 3.16), use of fibrinolytics during index hospitalisation (aOR $0.22,95 \%$ CI 0.10 to 0.48 ) became new predictors. In overall cohort comparing DAPT with clopidogrel and DAPT with prasugrel or ticagrelor, gender, admission for NSTEMI, and concurrent use of H2RA were no longer predictors in the 2014-2017 subgroup. Similar exclusion of predictors was obtained in the post-PCI cohorts when considering the same comparison in 2014-2017 subgroup. In medically treated ACS cohort comparing DAPT with clopidogrel and aspirin alone, chronic kidney 


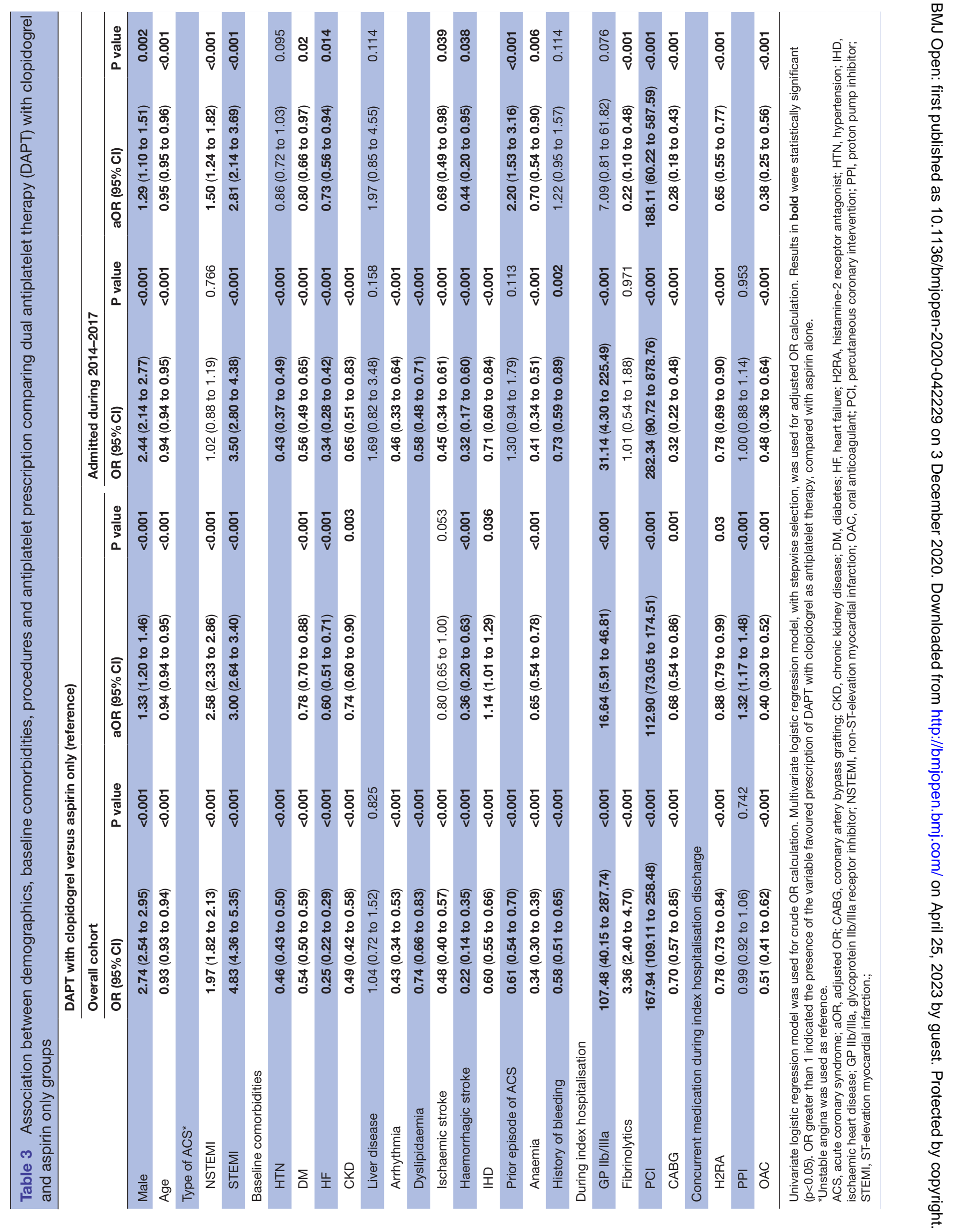




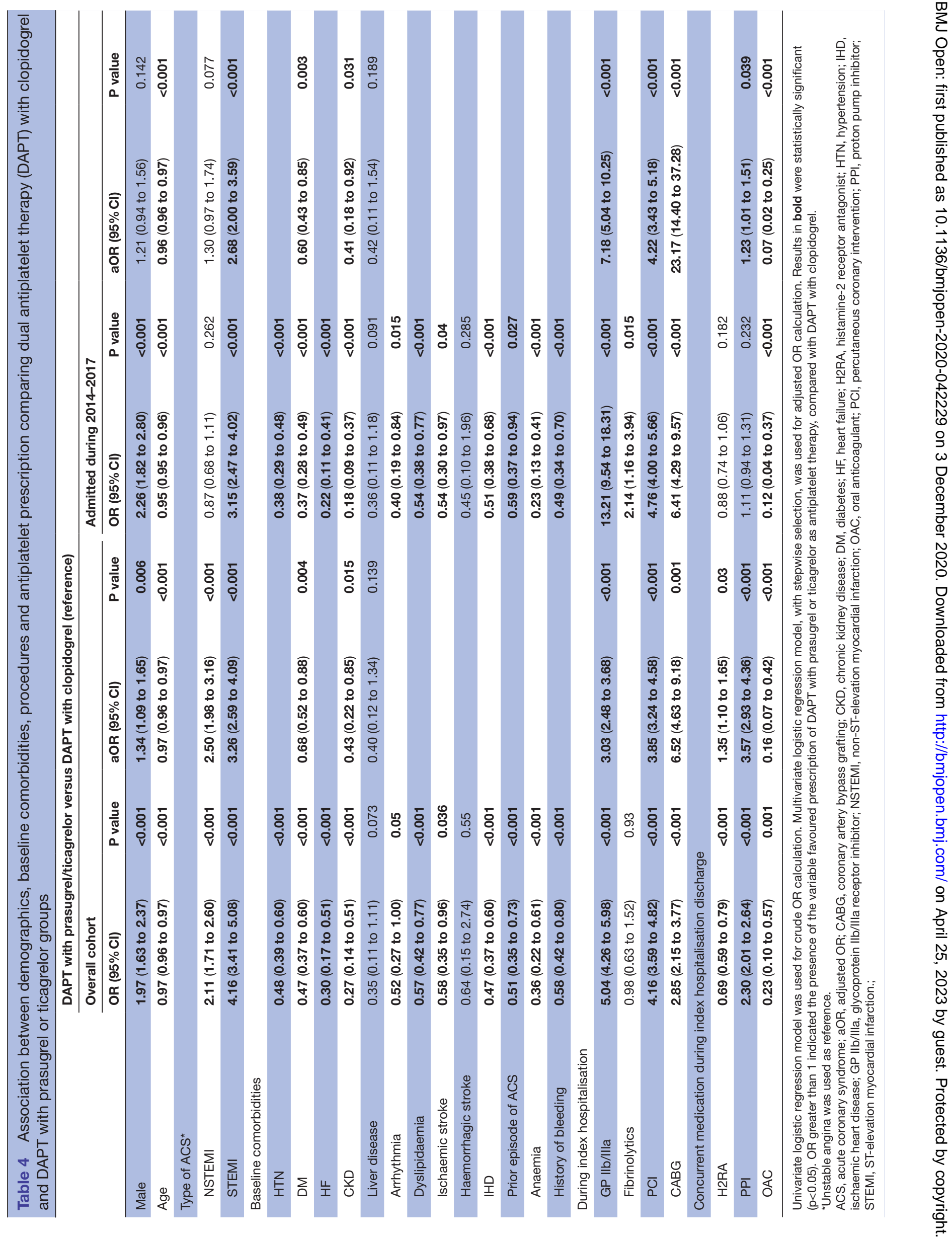




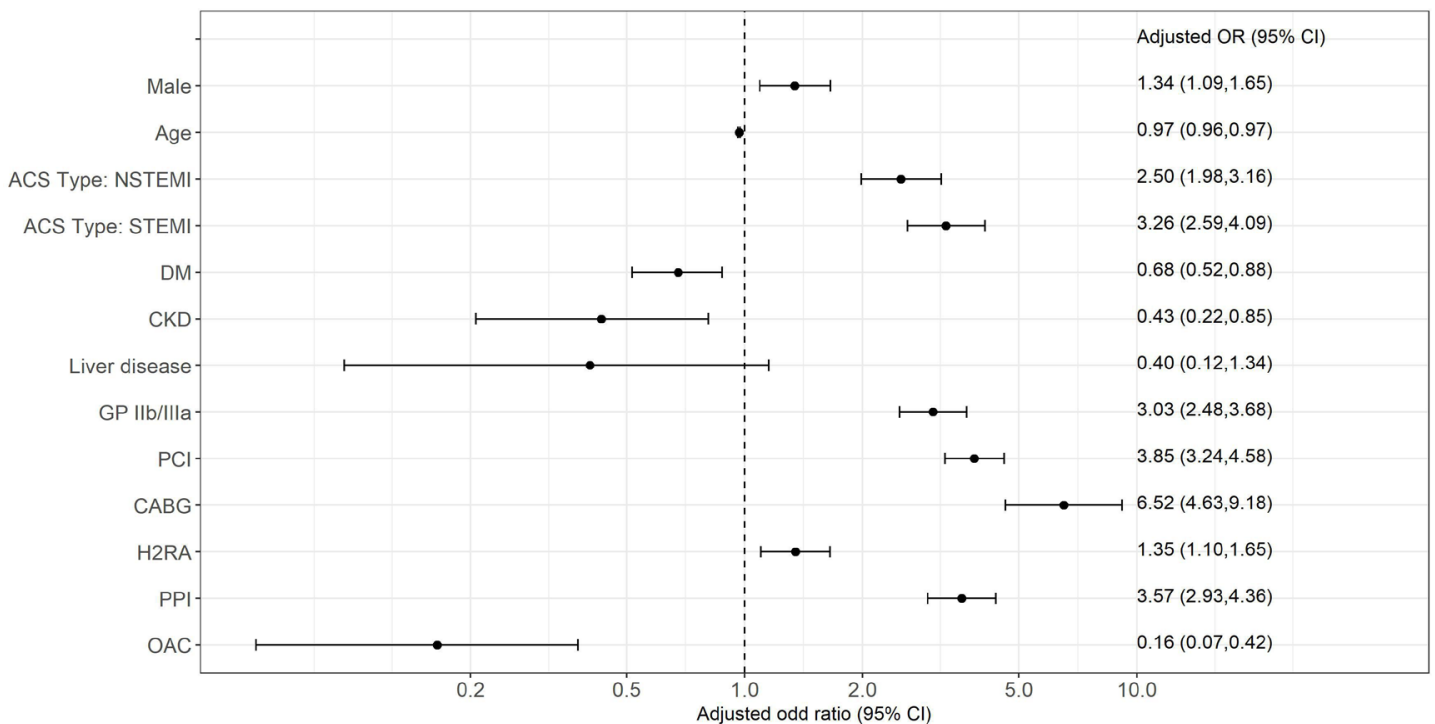

Figure 3 Adjusted ORs of dual antiplatelet therapy (DAPT) with prasugrel/ticagrelor prescription, compared with DAPT with clopidogrel. Adjusted OR was calculated with multivariate logistic regression. OR greater than 1 indicated that the presence of the variable was associated with prescription of DAPT with prasugrel or ticagrelor as antiplatelet therapy, compared with DAPT with clopidogrel. For acute coronary syndrome (ACS) type, unstable angina was used as reference. CABG, coronary artery bypass grafting; CKD, chronic kidney disease; DM, diabetes; GP Ilb/llla, glycoprotein Ilb/Illa receptor inhibitor; HF, heart failure; HTN, hypertension; H2RA, histamine-2 receptor antagonist; IHD, ischaemic heart disease; NSTEMI, non-ST-elevation myocardial infarction; OAC, oral anticoagulant; PCI, percutaneous coronary intervention; PPI, proton pump inhibitor; STEMI, ST-elevation myocardial infarction.

disease, use of GP IIb/IIIa and concurrent use of PPI were excluded as predictors, while prior episode of ACS (aOR 2.19, 95\% CI 1.53 to 3.15) and use of fibrinolytics (aOR $0.25,95 \%$ CI 0.11 to 0.56 ) were included as new predictors.

\section{DISCUSSION}

Thus far, there has not been a review on antiplatelet prescribing pattern in Hong Kong ACS population. This study is the first study to describe the antiplatelet use in Hong Kong patients with ACS during hospitalisation discharge for a 10-year review. An overall trend of increasing use of DAPT and decreasing use of aspirin alone in patients with ACS was observed, while proportion of ticagrelor use in DAPT was on rising trend over the 10-year study period. Prescription pattern of medication depended on the availability of medication, evidence of medication on disease management and recommendation from international professional bodies. Availability of medication and changes on guideline recommendation were examined along with the trend observed from our results.

In 2000, ACC/AHA guideline recommended use of aspirin, or clopidogrel in those unable to take aspirin, in patients with UA or NSTEMI. ${ }^{23}$ Subsequently in 2002, an update on the UA and NSTEMI guideline made an recommendation on addition of clopidogrel to aspirin in patients with whom non-interventional approach was planned..$^{24}$ Update on ACC/AHA guideline update for STEMI in 2004 also recommended use of clopidogrel in patients with planned PCI in addition to aspirin. ${ }^{25}$
In patients with PCI, ACC/AHA updated recommendation with Society for Cardiovascular Angiography and Interventions that both aspirin and clopidogrel should be given. ${ }^{26}$ Despite the fact that DAPT was advocated by the guideline recommendation before the start of study period, the use of DAPT was only increased from $33.7 \%$ in 2008 to $68.0 \%$ in 2017 in patients with ACS. Use of aspirin alone in patients with ACS decreased from 56.8\% in 2008 to $27.5 \%$ in 2017. In another words, there remained more than one-fourth of patients with ACS received aspirin only as antiplatelet treatment on index hospitalisation discharge. Vast majority of aspirin only group was medically treated without undergoing PCI $(99.6 \%)$ or CABG $(96.1 \%)$. Use of DAPT in post-PCI patients ranged from $96.9 \%$ to $98.1 \%$ during $2008-2012$ and remained at more than $99 \%$ since 2013. Overall DAPT utilisation in patients with ACS, particularly in medically treated subjects, was unsatisfactory.

Published studies on ACS antiplatelet prescription pattern revealed high adherence of DAPT in other countries. A study on antiplatelet use in Spain with data collected from multicentre registry demonstrated high adherence of DAPT in patients with ACS, ranging from $79 \%$ to $87 \%$ throughout the study period of $2012-2015 .{ }^{12}$ Yet such high adherence was not observed in other studies. For example, rate of DAPT in patients with ACS was ranging $67 \%-75 \%$ in Sweden and DAPT adherence rate in ACS subjects was $70.4 \%$ from Australia experience. ${ }^{11}$ When considering the trend of antiplatelet prescription pattern, study from Australia showed that DAPT use improved from $69.4 \%$ in 2013 to $78.4 \%$ in 
2016. ${ }^{11}$ Adherence rate of DAPT prescription in Hong Kong patients with ACS was lower than in other countries (average $46.8 \%$, ranging $33.7 \%-68.0 \%$ ).

Rate of DAPT use in medically treated patients with ACS increased gradually from $27.4 \%$ in 2008 to $54.5 \%$ in 2017 , while use of aspirin alone reduced from $62.4 \%$ in 2008 to $39.3 \%$ in 2012 . There remained $4.7 \%$ of medically treated patients with ACS discharged without any antiplatelet in 2017. It shall be noted that in medically treated patients with ACS, of which patients with NSTE-ACS shall be assumed from the point of guideline recommendation, DAPT should be used since hospitalisation and continued for at least 1 month.

Non-adherence of DAPT prescription in medically treated patients with ACS was observed in other studies. Experience in Australia during 2009-2016 revealed that in patients with ACS without undergoing PCI, 52.6\% of subjects received DAPT while $47.4 \%$ received single antiplatelet therapy. ${ }^{11}$ The Sweden study also showed that use of DAPT in patients with ACS without PCI improved from $44 \%$ in 2009 to $50 \%$ in $2013 .{ }^{10}$ Adherence to DAPT prescription in non-PCI ACS subjects was better in China, of which $81.8 \%$ of non-PCI NSTE-ACS subjects during 2014-2016 received DAPT. ${ }^{13}$ The Hong Kong data were comparable with the Caucasian experience, yet much lower than the China's experience (average 40.3\%, ranging $27.4 \%-54.5 \%$ ). The difference of DAPT prescription rate between China and Hong Kong could possible due to the dissimilarity of drug policy. National Essential Medicines List was established in China, of which enlisted medications could be reimbursed under medical insurance system to ensure availability and affordability of essential drugs. ${ }^{27}$ Clopidogrel had been enlisted in the National Essential Medicines Lists 2012 and there was not restriction on indication of drug, unlike the HA drug formulary in Hong Kong. ${ }^{28}$ Implementation of National Essential Medicines List was shown to improve rational use of drugs. ${ }^{27}$ The unrestricted use of P2Y12 inhibitors from the policy aspect may contribute to better prescription rate of DAPT in China than in Hong Kong.

When considering choice of P2Y12 receptor antagonist, guideline recommendation was updated accordingly with emerging evidence on prasugrel and ticagrelor as part of DAPT in ACS population. In 2013, ACCF/AHA guideline recommendation for management of STEMI suggested that in patients with STEMI undergoing primary PCI with stent insertion, aspirin should be given indefinitely, while P2Y12 receptor antagonist (clopidogrel, prasugrel or ticagrelor) should be given for 1 year for maintenance. ${ }^{6}$ Subsequently in 2014, AHA/ACC also updated their guidelines for management of NSTE-ACS on use of antiplatelet agents, suggesting that clopidogrel or ticagrelor should be used in addition to aspirin for up to 12 months in all patients with NSTE-ACS without contraindication, regardless of whether invasive revascularisation was used. ${ }^{5}$ It was also first mentioned in the 2014 ACC/AHA guidelines for NSTE-ACS that ticagrelor was preferred over clopidogrel. ${ }^{5}$ In 2016, ACC/AHA published another guideline focused on DAPT in patients with coronary artery disease. Medically treated patients with ACS without undergoing revascularisation were suggested to be treated with DAPT with either clopidogrel or ticagrelor for 12 months, while ticagrelor was preferred over clopidogrel. ${ }^{9}$ In post-PCI patients with ACS, DAPT with either clopidogrel, prasugrel or ticagrelor should be used. ${ }^{9}$ Prasugrel was preferred over clopidogrel, in patients who were not at high risk of bleeding and did not have history of stroke or transient ischaemic attack (TIA), whereas ticagrelor was preferred over clopidogrel. ${ }^{9}$

From our observation, use of DAPT with prasugrel in Hong Kong patients with ACS remained low. Use of DAPT with prasugrel was peaked at $2.9 \%$, when considering overall ACS cohort in 2014 and there was no record of prasugrel as discharge antiplatelet therapy in 2017. Use of DAPT with ticagrelor in patients with ACS increased from $2.7 \%$ in 2012 to $15.3 \%$ in 2017 . When focusing on proportional of ticagrelor use among three P2Y12 receptor antagonists, ticagrelor constituted $22.4 \%$ of post-ACS DAPT maintenance regimen in 2017, while the remaining portion were all receiving clopidogrel. Majority of patients receiving DAPT with ticagrelor underwent PCI during index hospitalisation $(71.4 \%)$. When considering post-PCI patients with ACS, use of DAPT with ticagrelor was increased from $6.9 \%$ in 2012 to $40.1 \%$ in 2017 , with an average rate of $17.9 \%$. Adherence of P2Y12 receptor antagonist in patients with post-ACS to international guideline recommendation on the preferred use of newer agent (ticagrelor) was, despite low, improving gradually.

Prevalence of use of prasugrel varied across studies. In Switzerland, use of prasugrel constituted $31.2 \%$ of antiplatelet regimen in post-PCI patients with ACS. ${ }^{29}$ Use of prasugrel in Spanish ACS population maintained at 8\%-14\% during 2012-2015, while experience in Belgium demonstrated use of prasugrel at $19.0 \%$ in patients with ACS. ${ }^{12}{ }^{14}$ Study in the USA showed that $18.4 \%$ of newer P2Y12 receptor antagonists users received prasugrel. ${ }^{30}$ Similar to Hong Kong data (average $0.8 \%$ ), use of prasugrel in Sweden was only $1 \%$ in post-PCI ACS subjects. ${ }^{10}$ While prasugrel was yet to be available in China, $10.6 \%$ of patients with acute MI in Korea during 2013-2015 were prescribed with prasugrel. ${ }^{13}{ }^{31}$ In Japan, use of prasugrel was common, accounting for $80.6 \%$ of P2Y12 inhibitor prescription in acute MI patients during 2015-2017, while majority of them received reduced dose of prasugrel at $3.75 \mathrm{mg}{ }^{32}$ Generally, low prevalence of prasugrel use could attribute to the increased bleeding and less net clinical benefit in the group of patients with history of stroke or transient ischaemic attack, age 75 years old or above, or those with body weight less than $60 \mathrm{~kg}$.

Use of ticagrelor in patients with ACS from other studies was more common as well. Prevalence of use of ticagrelor in Belgium study was $49.8 \%$ in ACS subjects. ${ }^{14}$ Spanish study demonstrated improved use of ticagrelor from $15 \%$ in 2012 to $28 \%$ in 2015 in patients with ACS. ${ }^{12}$ Results from both studies showed better prevalence on ticagrelor use in overall ACS population in other countries. 
When focusing in post-PCI patients with ACS, prevalence of DAPT with ticagrelor use was up to $54 \%$ from Swedish ACS nationwide registry, doubling that of DAPT with clopidogrel (26\%). ${ }^{10}$ Experience from Switzerland showed $39.3 \%$ of post-PCI patients with ACS received ticagrelor. ${ }^{29}$ It should be noted that there was also another $31.2 \%$ of patients receiving prasugrel, leading to a total of $70.5 \%$ of post-PCI patients with ACS receiving newer P2Y12 receptor antagonists, compared with $14.5 \%-40.1 \%$ of post-PCI patients with ACS receiving DAPT with prasugrel or ticagrelor in our study during 2012-2017. ${ }^{29}$ When considering Chinese population, use of DAPT with ticagrelor in post-PCI patients with NSTE-ACS was also low, reported at $11.1 \%{ }^{13}$ From these results, use of newer P2Y12 receptor antagonists was more conservative in Asia compared with Western countries. Asian has a lower body mass index and higher bleeding risk compared with Caucasian counterparts, leading to physicians' conservative attitude to prescribe newer P2Y12 receptor antagonists. ${ }^{334}$ Despite more use of DAPT with ticagrelor in patients with ACS in recent years, the results from our study showed that there was a delay of practice adaptation to the international guideline recommendation in Hong Kong.

Clinical benefit of newer P2Y12 receptor antagonists over clopidogrel was demonstrated in the landmark randomised controlled trials, TRITON-TIMI-38 study and PLATO study, and in the nationwide observational studies. ${ }^{785-37}$ Moreover, higher guideline adherence rate in patients with NSTE-ACS was associated with lower rate of MI or mortality. ${ }^{38}$ A 20-year nationwide observational study showed that the uptake of evidence-based procedural and medical intervention in patients with NSTEMI substantially reduced mortality and CV event. ${ }^{39}$ This highlights the importance of guideline adherence to patients' outcomes.

As we observed the consistently low adherence of DAPT, especially in DAPT with newer P2Y12 receptor antagonist, our study tried to illustrate the association of antiplatelet prescription and patients' demographics to explain the reason behind. Predictors for the aspirin only group compared with DAPT with clopidogrel included female, older age, diagnosis of UA (compared with NSTEMI and STEMI), diabetes, heart failure, prior history of stroke, anaemia, CABG, concurrent use of H2RA or OAC. Most of the predictors suggested that patients being more fragile were more likely to be prescribed with aspirin alone instead of DAPT. Similar result was demonstrated previously by Anastasius et al, that predictors for DAPT non-prescription in patients with ACS were CABG, use of warfarin, bleeding during hospitalisation, diagnosis of UA or NSTEMI (compared with STEMI), arrhythmia, hypertension and high Global Registry of Acute Coronary Events (GRACE) score. ${ }^{11}$ Our study also found that older age, diagnosis of UA (compared with STEMI), diabetes, absent of use of GP IIb/IIIa or PPI and concurrent use of OAC were the predictors of clopidogrel use compared with prasugrel or ticagrelor as part of DAPT. Our study detected less predictors than the published study. The Italian study demonstrated that those with clopidogrel prescription were more likely to be of older age, female, diabetes, renal failure, anaemia, atrial fibrillation, multivessel disease, worse Killip class, lower ejection fraction and higher GRACE and Can Rapid Risk Stratification of Unstable Angina Patients Suppress Adverse Outcomes with Early Implementation of the ACC/AHA Guidelines(CRUSADE) scores. ${ }^{40}$ Both studies suggested that patients receiving clopidogrel were generally more fragile and vulnerable, and thus it was postulated that physician tended to be more conservative when considering choice of medications.

Indeed, the above-mentioned findings on predictors for more conservative approach of antiplatelet therapy were consistent with the identified risk factors of bleeding in patients with ACS as mentioned from ACCF/AHA guideline on STEMI. ${ }^{6}$ Identified risk factors of bleeding included older age, female, heart failure, diabetes, history of gastrointestinal (GI) bleeding, anaemia, invasive strategy or use of chronic OAC. ${ }^{6}$ Female gender (OR 2.32, 95\% CI 1.98 to 2.72), increasing age (OR 1.17, 95\% CI 1.13 to 1.21 ), increased serum creatinine (OR $1.09,95 \%$ CI 1.07 to 1.12 ) and anaemia (OR $1.98,95 \%$ CI 1.65 to 2.37) were shown to be independent predictors for non-CABG related major bleeding. ${ }^{41}$ Major bleeding was shown to be associated with increased mortality. ${ }^{41} 42$ Therefore, the conservative choice of antiplatelet therapy could be due to increased risk of bleeding of patients with ACS.

There are a few limitations in our study. First, despite our study trying to match the availability of drug with the date of local registration, availability of newer medication within each institution could be not traced. The HA has established drug formulary with standardisation of drug policy since 2005 to ensure equal access of treatment for patients under the care of HA institutions. ${ }^{43}$ Yet decision on stocking of medications is based on the respective service needs for each individual institution. Therefore, availability of medication varies across different hospitals and clinics. This could cause unavailability of newer medication in certain hospital at earlier phase of study period. Second, choice of medication prescription does not depend merely on clinical evidence of medication or availability, but also affordability of medication by patients. Medications available within HA institution are classified into either general drug, special drug or self-financed item. For general drug and special drug prescribed with specified indications, medications would be provided at standard charges under HA institution. Yet for self-financed item and special drug prescribed with indication other than specified clinical condition, patients would be required to pay the full charge for the medications. ${ }^{43}$ Historical classification of study medications is unknown. Currently, all three P2Y12 receptor antagonists are under category of special drug. Prasugrel and ticagrelor are with the specified indication of post-PCI patients or alternative to hypersensitivity of clopidogrel, 
while clopidogrel are indicated for both post-coronary intervention and high-risk patients with ACS. Such difference of indication of P2Y12 receptor antagonists within HA drug formulary could lead to additional financial consideration of patients when choosing between clopidogrel and newer P2Y12 receptor antagonists. Third, the study relied on antiplatelet dispensing record in patients with ACS treated in institutions under HA. Prescribing pattern of antiplatelet therapy in private sector was not investigated; thus, this could not explicitly represent the whole landscape of antiplatelet agent prescribing pattern in Hong Kong. Fourth, the current study focused on antiplatelet therapy on patients' discharge. Use of antiplatelet during index hospitalisation was not captured. There could be chance that patients were prescribed otherwise, yet with the bleeding episode, more conservative antiplatelet approach was adopted at discharge. Finally, the cross-sectional cohort design of the study did not consider the subsequent follow-up of antiplatelet treatment. Any changes on antiplatelet therapy could not be captured, and thus the study could not reflect the overall antiplatelet management of patients with ACS.

\section{CONCLUSION}

The trend of prescription pattern of antiplatelet therapy in patients with ACS was evaluated using real-world territory-wide electronic database in Hong Kong. Use of DAPT and newer P2Y12 receptor antagonists in patients with ACS was suboptimal yet improving. A delay of practice adaptation to the international guideline recommendation in Hong Kong was observed. Predictors for more conservative approach of antiplatelet treatment in patients with ACS were examined, of which the results were generally consistent with the guideline identified risk factors for bleeding risk.

Contributors VWL and ASL designed the study. VWL and BPY assisted in data acquisition logistics, reviewed and commented the article. ASL collected and analysed the data and drafted the article.

Funding The authors have not declared a specific grant for this research from any funding agency in the public, commercial or not-for-profit sectors.

Competing interests None declared.

Patient consent for publication Not required.

Ethics approval Ethics approval was obtained from the Joint Chinese University of Hong Kong - NTEC Clinical Research Ethics Committee (CREC no.: 2019.090).

Provenance and peer review Not commissioned; externally peer reviewed.

Data availability statement Data may be obtained from Hospital Authority Hong Kong and are not publicly available.

Supplemental material This content has been supplied by the author(s). It has not been vetted by BMJ Publishing Group Limited (BMJ) and may not have been peer-reviewed. Any opinions or recommendations discussed are solely those of the author(s) and are not endorsed by BMJ. BMJ disclaims all liability and responsibility arising from any reliance placed on the content. Where the content includes any translated material, BMJ does not warrant the accuracy and reliability of the translations (including but not limited to local regulations, clinical guidelines, terminology, drug names and drug dosages), and is not responsible for any error and/or omissions arising from translation and adaptation or otherwise.

Open access This is an open access article distributed in accordance with the Creative Commons Attribution Non Commercial (CC BY-NC 4.0) license, which permits others to distribute, remix, adapt, build upon this work non-commercially, and license their derivative works on different terms, provided the original work is properly cited, appropriate credit is given, any changes made indicated, and the use is non-commercial. See: http://creativecommons.org/licenses/by-nc/4.0/.

\section{ORCID iDs}

Amy SM Lam http://orcid.org/0000-0003-0139-2328

Vivian WY Lee http://orcid.org/0000-0001-5802-8899

\section{REFERENCES}

1 Yusuf S, Zhao F, Mehta SR, et al. Effects of clopidogrel in addition to aspirin in patients with acute coronary syndromes without STsegment elevation. N Engl J Med 2001;345:494-502.

2 Mehta SR, Yusuf S, Peters RJ, et al. Effects of pretreatment with clopidogrel and aspirin followed by long-term therapy in patients undergoing percutaneous coronary intervention: the PCI-CURE study. Lancet 2001;358:527-33.

3 Steinhubl SR, Berger PB, Mann JT, et al. Early and sustained dual oral antiplatelet therapy following percutaneous coronary intervention: a randomized controlled trial. JAMA 2002;288:2411-20.

4 Sabatine MS, Cannon CP, Gibson CM, et al. Addition of clopidogrel to aspirin and fibrinolytic therapy for myocardial infarction with STsegment elevation. N Engl J Med 2005;352:1179-89.

5 Amsterdam EA, Wenger NK, Brindis RG, et al. 2014 AHA/ACC guideline for the management of patients with non-ST-elevation acute coronary syndromes: a report of the American College of Cardiology/American heart association Task force on practice guidelines. J Am Coll Cardiol 2014;64:e139-228.

6 O'Gara PT, Kushner FG, Ascheim DD, et al. 2013 ACCF/AHA guideline for the management of ST-elevation myocardial infarction: a report of the American College of cardiology Foundation/American heart association Task force on practice guidelines. Circulation 2013;127:e362-425.

7 Wiviott SD, Braunwald E, McCabe $\mathrm{CH}$, et al. Prasugrel versus clopidogrel in patients with acute coronary syndromes. N Engl J Med 2007;357:2001-15.

8 Wallentin L, Becker RC, Budaj A, et al. Ticagrelor versus clopidogrel in patients with acute coronary syndromes. $N$ Engl $\mathrm{J}$ Med 2009;361:1045-57.

9 Levine GN, Bates ER, Bittl JA, et al. 2016 ACC/AHA guideline focused update on duration of dual antiplatelet therapy in patients with coronary artery disease: a report of the American College of Cardiology/American heart association Task force on clinical practice guidelines. J Am Coll Cardiol 2016;68:1082-115.

10 Angerås $\mathrm{O}$, Hasvold $\mathrm{P}$, Thuresson $\mathrm{M}$, et al. Treatment pattern of contemporary dual antiplatelet therapies after acute coronary syndrome: a Swedish nationwide population-based cohort study. Scand Cardiovasc J 2016;50:99-107.

11 Anastasius M, Lau JK, Hyun K, et al. The underutilisation of dual antiplatelet therapy in acute coronary syndrome. Int $J$ Cardiol 2017;240:30-6.

12 Esteve-Pastor MA, Ruíz-Nodar JM, Orenes-Piñero E, et al. Temporal trends in the use of antiplatelet therapy in patients with acute coronary syndromes. J Cardiovasc Pharmacol Ther 2018;23:57-65.

13 Yang Q, Wang Y, Liu J, et al. Invasive management strategies and antithrombotic treatments in patients with Non-ST-SegmentElevation acute coronary syndrome in China: findings from the improving CCC project (care for cardiovascular disease in China). Circ Cardiovasc Interv 2017;10:e004750.

14 Claeys MJ, Beauloye C, Pourbaix S, et al. Real world insights on the initiation and treatment duration of oral antiplatelets in acute coronary syndromes: a retrospective cohort study. Eur Heart $J$ Cardiovasc Pharmacother 2017;3:189-97.

15 Hospital Authority. Introduction. Available: https://www3.ha.org.hk/ ntec/content/background_e.asp [Accessed 25 Jun 2020].

16 Department of Health HKSAR. Health facts of Hong Kong 2018 edition, 2018.

17 Hospital Authority. Strategic service framework for coronary heart disease; 2013.

18 Sing C-W, Woo Y-C, Lee ACH, et al. Validity of major osteoporotic fracture diagnosis codes in the clinical data analysis and reporting system in Hong Kong. Pharmacoepidemiol Drug Saf 2017;26:973-6.

19 Lip GYH, Collet J-P, Haude M, et al. 2018 joint European consensus document on the management of antithrombotic therapy in atrial fibrillation patients presenting with acute coronary syndrome and/ or undergoing percutaneous cardiovascular interventions: a joint consensus document of the European heart rhythm association (EHRA), European Society of cardiology Working group on 
thrombosis, European association of percutaneous cardiovascular interventions (EAPCI), and European association of acute cardiac care (ACCA) endorsed by the heart rhythm Society (HRS), AsiaPacific heart rhythm Society (APHRS), Latin America heart rhythm Society (LAHRS), and cardiac arrhythmia Society of southern Africa (CASSA). Europace 2019;21:192-3.

20 Sørensen R, Hansen ML, Abildstrom SZ, et al. Risk of bleeding in patients with acute myocardial infarction treated with different combinations of aspirin, clopidogrel, and vitamin $\mathrm{K}$ antagonists in Denmark: a retrospective analysis of nationwide registry data. Lancet 2009;374:1967-74.

21 Jneid H, Anderson JL, Wright RS, et al. 2012 ACCF/AHA focused update of the Guideline for the management of patients with unstable angina/non-ST-elevation myocardial infarction (updating the 2007 guideline and replacing the 2011 focused update): a report of the American College of cardiology Foundation/American heart association Task force on practice guidelines. J Am Coll Cardiol 2012;60:645-81.

$22 \mathrm{Kim} \mathrm{K}$, Lee TA, Touchette DR, et al. Contemporary trends in oral antiplatelet agent use in patients treated with percutaneous coronary intervention for acute coronary syndrome. J Manag Care Spec Pharm 2017;23:57-63.

23 Braunwald E, Antman EM, Beasley JW, et al. ACC/AHA guidelines for the management of patients with unstable angina and non-STsegment elevation myocardial infarction. A report of the American College of Cardiology/American heart association Task force on practice guidelines (Committee on the management of patients with unstable angina). J Am Coll Cardiol 2000;36:970-1062.

24 Braunwald E, Antman EM, Beasley JW, et al. ACC/AHA 2002 guideline update for the management of patients with unstable angina and non-ST-segment elevation myocardial infarction-summary article: a report of the American College of Cardiology/ American Heart Association task force on practice guidelines (Committee on the Management of Patients With Unstable Angina). J Am Coll Cardiol 2002;40:1366-74.

25 Antman EM, Anbe DT, Armstrong PW, et al. ACC/AHA guidelines for the management of patients with ST-elevation myocardial infarction-executive summary. A report of the American College of Cardiology/ American Heart Association Task Force on Practice Guidelines (Writing Committee to revise the 1999 guidelines for the management of patients with acute myocardial infarction). J Am Coll Cardiol 2004:44:671-719.

26 Smith SC, Feldman TE, Hirshfeld JW, et al. ACC/AHA/SCAI 2005 guideline update for percutaneous coronary Intervention-Summary article: a report of the American College of Cardiology/American heart association Task force on practice guidelines (ACC/AHA/SCAI writing Committee to update the 2001 guidelines for percutaneous coronary intervention). J Am Coll Cardiol 2006;47:216-35.

27 Tao W, Zeng Z, Dang H, et al. Towards universal health coverage: achievements and challenges of 10 years of healthcare reform in China. BMJ Glob Health 2020;5:e002087.

28 National Medical Products Administration. National essential medicines Lists 2012, 2012.

29 Zanchin T, Temperli F, Karagiannis A, et al. Frequency, reasons, and impact of premature ticagrelor discontinuation in patients undergoing coronary revascularization in routine clinical practice: results from the
Bern percutaneous coronary intervention registry. Circ Cardiovasc Interv 2018;11:e006132.

30 Larmore C, Effron MB, Molife C, et al. "Real-World" Comparison of Prasugrel With Ticagrelor in Patients With Acute Coronary Syndrome Treated With Percutaneous Coronary Intervention in the United States. Catheter Cardiovasc Interv 2016;88:535-44.

31 Kim C, Shin DH, Ahn CM, et al. The use pattern and clinical impact of new antiplatelet agents including prasugrel and ticagrelor on 30-day outcomes after acute myocardial infarction in Korea: Korean health insurance review and assessment data. Korean Circ $J$ 2017;47:888-97.

32 Honda S, Nishihira K, Kojima S, et al. Rationale, design, and baseline characteristics of the prospective Japan acute myocardial infarction registry (JAMIR). Cardiovasc Drugs Ther 2019;33:97-103.

33 Huo $\mathrm{Y}$, Jeong $\mathrm{Y}-\mathrm{H}$, Gong $\mathrm{Y}$, et al. 2018 update of expert consensus statement on antiplatelet therapy in East Asian patients with ACS or undergoing PCI. Sci Bull 2019;64:166-79.

34 Tscharre M, Egger F, Machata M, et al. Contemporary use of P2Y12inhibitors in patients with acute coronary syndrome undergoing percutaneous coronary intervention in Austria: a prospective, multicentre registry. PLoS One 2017;12:e0179349.

35 Sahlén A, Varenhorst C, Lagerqvist B, et al. Outcomes in patients treated with ticagrelor or clopidogrel after acute myocardial infarction: experiences from SWEDEHEART registry. Eur Heart $J$ 2016;37:3335-42.

36 Choe JC, Cha KS, Ahn J, et al. Comparison of prescription rates and clinical outcomes in acute coronary syndrome patients who underwent percutaneous coronary intervention using different P2Y $Y_{12}$ inhibitors in a large observational study. Int J Cardiol 2019;274:21-6.

37 Yun JE, Kim YJ, Park JJ, et al. Safety and Effectiveness of Contemporary P2Y ${ }_{12}$ Inhibitors in an East Asian Population With Acute Coronary Syndrome: A Nationwide Population-Based Cohort Study. J Am Heart Assoc 2019;8:e012078.

38 Engel J, Damen NL, van der Wulp I, et al. Adherence to cardiac practice guidelines in the management of non-ST-elevation acute coronary syndromes: a systematic literature review. Curr Cardiol Rev 2017;13:3-27.

39 Szummer K, Wallentin L, Lindhagen L, et al. Relations between implementation of new treatments and improved outcomes in patients with non-ST-elevation myocardial infarction during the last 20 years: experiences from SWEDEHEART registry 1995 to 2014. Eur Heart J 2018;39:3766-76.

40 Castini D, Persampieri S, Cazzaniga S, et al. Real-world clopidogrel utilization in acute coronary syndromes: patients selection and outcomes in a single-center experience. Ther Adv Cardiovasc Dis 2017;11:323-31.

41 Mehran R, Pocock SJ, Nikolsky E, et al. A risk score to predict bleeding in patients with acute coronary syndromes. J Am Coll Cardiol 2010;55:2556-66.

42 Eikelboom JW, Mehta SR, Anand SS, et al. Adverse impact of bleeding on prognosis in patients with acute coronary syndromes. Circulation 2006;114:774-82.

43 Hospital Authority. Drug formulary. Available: https://www.ha.org. $\mathrm{hk} / \mathrm{hadf} / \mathrm{en}-\mathrm{us} /$ Updated-HA-Drug-Formulary/Drug-Formulary.html [Accessed 26 May 2020]. 